\title{
Integrating Metta Into CBT: How Loving Kindness and Compassion Meditation Can Enhance CBT for Treating Anxiety and Depression
}

\author{
Simona Stefan ${ }^{\text {ab }}$, Stefan G. Hofmann ${ }^{\mathrm{a}}$ \\ [a] Boston University, Department of Psychological and Brain Sciences, Boston, MA, USA. [b] Department of Clinical \\ Psychology and Psychotherapy, Babes-Bolyai University, Cluj-Napoca, Romania.
}

Clinical Psychology in Europe, 2019, Vol. 1(3), Article e32941, https://doi.org/10.32872/cpe.v1i3.32941

Received: 2019-01-08 • Accepted: 2019-06-11 • Published (VoR): 2019-09-20

Handling Editor: Cornelia Weise, Philipps-University of Marburg, Marburg, Germany

Corresponding Author: Stefan G. Hofmann, 900 Commonwealth Avenue, Boston, MA, USA. E-mail: shofmann@bu.edu

\section{Abstract}

Background: Loving kindness meditation and compassion meditation are traditional Buddhist practices that have recently been introduced and investigated in psychotherapy with promising results. Both meditation practices emphasize metta, a mental state of positive energy and kindness towards oneself and other beings, as opposed to the anger, hostility, or self-loathing that often accompany emotional problems.

Method: We conducted a qualitative review of the literature to produce an integrative review.

Results: Metta meditation appears to be particularly useful for treating depression and social anxiety, both characterized by low positive affect and negative attitudes and core beliefs about the self.

Conclusion: Metta meditation can aid therapy by promoting more adaptive self-images, social connectedness, and emotional experiences.

\section{Keywords}

meditation, CBT, depression, anxiety, loving kindness, compassion 


\section{Highlights}

- Loving kindness and compassion meditation (metta) have been recently introduced in psychotherapy.

- Metta addresses shame, anger, and hostility, and promotes an accepting attitude towards oneself.

- Metta meditation increases positive affect.

- Metta meditation can be particularly useful in social anxiety and depression.

\section{Loving Kindness and Compassion Meditation in Psychotherapy}

Initially derived from Buddhist practices, the concept of mindfulness, briefly defined as the non-judgmental, accepting experience of the present, as it unfolds moment by moment, has nowadays become ubiquitous in the fields of psychotherapy and mental health, as well as in self-help and popular psychology. Psychotherapy interventions, such as Mindfulness-Based Stress Reduction (MBSR; [Kabat-Zinn, 1982] and Mindfulness Based Cognitive Therapy (MBST; [Segal, Williams, \& Teasdale, 2002] are considered established interventions for conditions such as chronic stress and depression (Hofmann, Sawyer, Witt, \& Oh, 2010). Furthermore, mindfulness has become an integral part of various psychological interventions, such as Acceptance and Commitment Therapy (ACT; Hayes, 2004) and Dialectical Behavioral Therapy (DBT; Linehan et al., 1999). More recently, other forms of meditation inspired by Buddhist philosophy, especially loving kindness and compassion meditation, have been introduced and investigated in mental health interventions (Hofmann, Grossman, \& Hinton, 2011; Zeng, Chiu, Wang, Oei, \& Leung, 2015). Although the term was originally associated with loving kindness, we will refer to both as metta interventions, as both types of interventions instill a sense of positive energy ( metta) directed at oneself and other beings. Taking a mindful stance, they further encourage a kinder view on oneself and others, which is central to addressing many emotional intra and interpersonal problems (e.g., anger, hostility, depression, anxiety). Due to the warmth and sense of connection they provide, metta interventions increase positive affect, particularly emotions related to calmness and safety. In this article, we will explore how metta-derived practices can help build a healthier view and a warmer attitude towards oneself, and how we can combine them with more traditional intervention techniques, specifically cognitive-behavioral therapy (CBT), when treating depression and social anxiety.

According to the Buddhist tradition, loving kindness (metta) and compassion (karuna) are two of the four brahma viharas, or sublime states, which also include sympathetic joy (mutida; feeling joy when others are joyful) and equanimity (upekkha; tranquility, equidistance, calmness) (Hofmann et al., 2011). They are centered on the idea of universal and 
unconditional kindness and interconnection among human beings, as opposed to the harshness and isolation which often accompany the experience of emotional suffering. Whereas loving kindness meditation promotes an attitude of warmth and positive energy directed at oneself and all other beings, compassion entails the drive and commitment to alleviate suffering (Graser \& Stangier, 2018). Thus, compared to loving kindness, the experience of compassion encompasses a warm feeling of sadness. Self-compassion (i.e., an attitude of compassion directed towards the self), as defined by (Neff, 2003) has emerged as a somewhat separate concept made up of three components: mindfulness (as opposed to over-identifying with one's own suffering), common humanity (as opposed to isolation), and self-kindness (a kind attitude towards the self, as opposed to harshness and self-criticism). An alternative conceptualization promoted by Gilbert describes compassion (and self-compassion implicitly) as a positive affect motivational system, with evolutionary roots and specific neurobiological underpinnings, channeled on soothing and providing care, safety, and empathy (Gilbert, 2005; MacBeth \& Gumley, 2012). Studies have shown self-compassion to be consistently inversely related to psychopathology measures (e.g., depression, anxiety, stress), pointing to its potential role in preventing negative, dysfunctional emotions (see MacBeth \& Gumley, 2012).

In practice, loving kindness meditation (LKM) involves the mental repetition of phrases directed at others' and one's well-being and relief from suffering, in a non-judgmental and observing mental stance (e.g., "may you be well”, "may you be happy", "may we be safe"). As LKM progresses, kindness is directed towards more and more challenging recipients, starting with oneself or a friend, continuing with a neutral person, and ending with the entire universe (Hofmann et al., 2011). The purpose is to experience a wish for universal well-being with a kind and tender mindset. Compassion meditation is similar to LKM, but encompasses the acknowledgement of suffering (e.g., "this is a moment of suffering"), recognizing the communality of suffering (e.g., "suffering is a part of life) and committing to a position of kindness to oneself or others (e.g., "may I be kind to myself"), often accompanied by compassionate imagery - imagining a compassionate person, character, or any other entity evoking features of wisdom, empathy, and understanding (Gilbert \& Procter, 2006; Neff, 2011). However, there are multiple techniques for delivering LKM and compassion meditation (see Finlay-Jones, 2017 for a review) and many exercises actually combine the two, generating some conceptual overlap in the field (Shonin, Van Gordon, Compare, Zangeneh, \& Griffiths, 2015).

So far, previous results have shown that LKM and compassion meditation interventions are effective in reducing depression, and increasing mindfulness, compassion, and self-compassion (against passive control conditions), as well as positive emotions (against relaxation) (Galante, Galante, Bekkers, \& Gallacher, 2014). Evidence suggests that these meditation interventions are useful for both clinical and healthy populations, in addressing psychological distress, positive and negative affect, the frequency and intensity of positive thoughts and emotions, interpersonal skills, and empathic accuracy (Shonin et 
al., 2015). A recent review (Graser \& Stangier, 2018) also evidenced that, looking at randomized trials only, compassion-based interventions are effective for psychotic disorders, depression, eating disorders, and patients with suicide attempts, while loving kindness interventions are effective in treating chronic pain, and a combination of both is useful for borderline personality disorder. Still, there are few randomized trials and even fewer that compared compassion and loving kindness interventions with active control conditions. Therefore, it is not certain whether these related strategies bring a unique, unshared contribution to relieving distress or promoting positive affect. Also, results are difficult to summarize because of the divergent conceptualizations of metta interventions; using very similar terminology, studies refer to compassion and/or loving kindness interventions as 1 . Single sessions consisting of brief exercises (e.g., Feldman, Greeson, \& Senville, 2010), 2. Several sessions of meditation (e.g., Carson et al., 2005; Hofmann et al., 2015), 3. Specific interventions which include, but are not restricted to compassion/loving kindness meditation, such as compassion-focused therapy (e.g., Gilbert \& Procter, 2006) or cognitively-based compassion training (e.g., Mascaro, Rilling, Tenzin Negi, \& Raison, 2013), or 4. Combining these interventions with CBT (e.g., Beaumont, Galpin, \& Jenkins, 2012).

\section{Loving Kindness and Self-Compassion in Depression and Social Anxiety Disorder}

\section{Negative Self-Views, Self-Criticism, and Shame in Depression and Social Anxiety}

Conceptually, due to their focus on promoting a kind, accepting view of oneself and others, these interventions particularly resonate with disorders characterized by self-criticism and shame (Gilbert \& Procter, 2006), anger, and hostility (Hofmann et al., 2011). The experience of shame and self-criticism is transdiagnostic, prevalent in disorders such as depression, social anxiety disorder, psychotic disorders, PTSD, eating disorders, and personality disorders (Gilbert, Pehl, \& Allan, 1994; Thompson \& Waltz, 2008).

For instance, in depression, the classical cognitive-behavioral model (Beck \& Alford, 2009) emphasizes the role of negative core beliefs related to oneself (worthlessness/ helplessness, unlovability), which further lead to a strain of negative automatic thoughts supporting the depressed, negative affect. Depressed individuals thus often have a harsh, critical attitude towards themselves, which is difficult to change. In this sense, studies have found that self-coldness (i.e., the reverse of self-compassion, including self-judgment, isolation, and over-identification) is a strong predictor of depressive symptoms in the general population both cross-sectional and longitudinally, over a period of 1 year (López, Sanderman, \& Schroevers, 2018). Also, depressed outpatients score lower on selfcompassion as compared to never-depressed participants, even when controlling for de- 
pression levels, with symptom-focused rumination and behavioral avoidance mediating the relation between self-compassion and depression (Krieger, Altenstein, Baettig, Doerig, \& Holtforth, 2013). Self-compassion also predicts subsequent depressive symptoms in clinical patients, while depression does not predict self-compassion (Krieger, Berger, \& Holtforth, 2016), and the relation between self-compassion and depression appears to be mediated by emotion regulation skills (Diedrich, Burger, Kirchner, \& Berking, 2017). Additionally, personality traits such as dependency (the tendency to rely excessively on other people and their approval) and self-criticism are related to depression severity scores in clinical (Luyten et al., 2007) and remitted depressives (Mongrain \& Leather, 2006), and constitute independent predictors (Luyten et al., 2007).

Similarly, socially anxious individuals display low self-esteem, high self-criticism, and dependency, with self-criticism as the strongest predictor of social anxiety symptoms (Iancu, Bodner, \& Ben-Zion, 2015). Also, people with social anxiety disorder show lower levels of self-compassion when compared to healthy controls, and within group, selfcompassion is related to fear of negative and positive evaluation (Werner et al., 2012). Additionally, shame and shame-proneness (the tendency to experience shame frequently) are particularly important in social anxiety disorder, being associated with social anxiety symptoms even after controlling for levels of depression and guilt (Fergus, Valentiner, McGrath, \& Jencius, 2010). Following psychological intervention, changes in social anxiety symptoms are further associated with decreases in shame proneness (Fergus et al., 2010).

\section{Emotion Regulation and Negative Self-Schemas}

Emotions can be regulated intrapersonally (Hofmann, Sawyer, Fang, \& Asnaani, 2012) or interpersonally (Hofmann, 2014; Hofmann \& Doan, 2018). Strategies that involve other people are interpersonal emotion regulation and include strategies such as soothing and social modeling (Hofmann, Carpenter, \& Curtiss, 2016). These strategies appear to enhance emotions by targeting the social self (Hofmann \& Doan, 2018). An example of an intrapersonal emotion regulation is cognitive reappraisal (e.g., Hofmann, 2016), a strategy aimed to cognitively modify one's perspective as to elicit an alternative emotional response (as conducted in the process of cognitive restructuring). It is usually less effectively used by currently depressed participants (Visted, Vøllestad, Nielsen, \& Schanche, 2018). Possibly, this is because cognitive reappraisal is demanding on the executive functions, which are sometimes impaired in depression, or because cognitive change is incongruent with the depressive mood and its subsequent negative and ruminative thinking style (Gotlib \& Joormann, 2010). Alternative accounts posit that depressive thinking is hard to change because although patients may logically understand that their thinking is distorted, they cannot embrace kinder, healthier views of themselves if they lack the emotional experience of being cared for. Many depressed patients report having been abused, bullied, or heavily criticized during childhood, and experienced little parental warmth. Self- 
criticism thus becomes a sort of inner voice, towards which the patient often assumes a submissive position (Gilbert, Clarke, Hempel, Miles, \& Irons, 2004). Therefore, in these cases, the motivational system related to soothing and safeness appears to be "malfunctioning". Usually, the activation of the soothing system (i.e. prompted by affection and safety signals perceived from others or oneself) deactivates defensive emotions (e.g., anxiety) and behaviors, and also turns off behaviors related to goal seeking, achieving, and acquiring, instead eliciting a state of calmness and connectedness. However, with depressed individuals, this system seems to be suppressed, possibly because its development was impaired at critical times in the past (Gilbert, 2005). In other words, depressed people have difficulty soothing themselves because the experience of being cared for is affectively foreign. This is why metta interventions could be particularly useful, since they do not aim to merely restructure negative self-views, but to create an inner experience of warmth and peace by promoting a qualitatively different kind of attitude towards oneself. Furthermore, self-criticism is highly prevalent in social anxiety disorder compared to other anxiety disorders, and remains at elevated levels even in people with history of social anxiety only (Cox, Fleet, \& Stein, 2004). Also, people with social anxiety often have high levels of perfectionism and unrealistic social standards (Hofmann, 2007), intrusive self-deprecating thoughts, and hostility and paranoia (Hofmann \& Otto, 2018), which combine with poor cognitive and emotion regulation strategies in the face of perceived threats and challenges, further complicating treatment (Flett \& Hewitt, 2014). Cognitive models of social anxiety disorder also emphasize maladaptive self-beliefs (Clark \& Wells, 1995; Farmer, Kashdan, \& Weeks, 2014) as central to symptom development, like high standard self-beliefs, conditional self-beliefs (e.g., "If people see I'm anxious, they'll think badly of me"), and unconditional beliefs (e.g., "I'm weak"). Similarly to depressed patients, individuals with social anxiety disorder also have diminished levels of positive affect due to lack of normative positive biases, unusual processing of positive events in the form of dampening positive affect, and lack of positive self-evaluations, which also contribute to living in a cold, harsh inner world (Farmer et al., 2014).

\section{Efficacy of Metta Interventions in Depression and Social Anxiety. Can We Change the Inner Experience (Qualia) in Relation to Oneself?}

Compassion-based (metta) interventions have been found to be effective in treating depression, anxiety, and shame, although the conceptualizations and treatment approaches are diverse (Finlay-Jones, 2017; Kirby, 2017). For instance, compassion-focused therapy (Gilbert \& Procter, 2006) is itself a form of psychotherapy, including the functional analysis of self-criticism and safety behaviors, explicitly training clients in decentering from their inner self-critical voice, and using experiential techniques such as compassion imagery, compassionate letter writing, or the two-chair technique. Compassion-focused therapy has been found to be effective in increasing happiness and mindfulness and de- 
creasing worry and emotional suppression in the general population (Jazaieri et al., 2014), as well as in schizophrenic, anxious, depressed, and disordered eating populations (see Graser \& Stangier, 2018 and Kirby, 2017). Another type of compassion intervention, mindfulness-based compassionate living was administered online and was found to be effective in reducing depressive and anxiety symptoms in participants with high levels of self-criticism, thus pointing to its potential as a transdiagnostic intervention (Krieger et al., 2019).

As an emotion regulation strategy, self-compassion is similarly effective as reappraisal and acceptance in reducing depressed mood following a mood induction task in depressed participants, but the effect seems to be moderated by baseline levels of depression, in the sense that self-compassion appears to be more effective than reappraisal for more severely depressed participants (Diedrich, Grant, Hofmann, Hiller, \& Berking, 2014). Also, even a short, 7-minute LKM exercise (i.e., imagining two loved ones sending their love to the participants) can increase explicit and implicit positivity towards strangers and implicit positivity towards the self, as well as positive affect (calm, happy, loving) when compared to a control condition (Hutcherson, Seppala, \& Gross, 2008).

Regarding social anxiety, a recent study (Cândea \& Szentágotai-Tătar, 2018) examined self-compassion as an emotion regulation strategy (i.e., for people with social anxiety) comparing self-compassion to cognitive reappraisal and waitlist. They found no differences at posttest between the groups, although the self-compassion group had significantly lower levels of shame-proneness and fear of negative evaluation at posttest compared to the pretest levels. Also, shame-proneness decreased from pre to posttest only in the selfcompassion and cognitive reappraisal groups. Similar results had been previously obtained, showing that self-compassion and cognitive reappraisal as emotion regulation strategies are similarly effective in reducing self-conscious negative emotions (a combination of shame, embarrassment, shyness, guilt, and regret), and more effective than responsibility reattribution and control condition (Arimitsu \& Hofmann, 2017). All these results suggest that metta interventions can qualitatively change the inner experience of oneself, thus fostering feelings of warmth, acceptance, and peace.

\section{Positive Affect and Its Role in Depression and Social Anxiety: How Metta Interventions Can Help}

The data previously presented suggest that being overly self-critical and exhibiting an inflexible vision of oneself are at the core of depression and social anxiety disorder. Therefore, metta-focused interventions may be particularly beneficial for treating these problems. According to the emotion dysregulation model of mood and anxiety disorders (Hofmann et al., 2012), apart from the enduring negative affect, both depression and social anxiety are characterized by deficiencies in positive affect (Brown, 2007), which are less specifically targeted by CBT protocols, potentially with the exception of behavioral activation. Increasing positive affect is especially important in depression and social 
anxiety since, apart from increasing well-being as proposed by the broaden and build theory (Fredrickson, 2001), it enhances behavioral repertoires, promoting approach behaviors to relevant situations (e.g., social situations, pleasant activities, new contexts and challenges), instead of the typical withdrawal and avoidance behaviors. Essentially, positive emotions extend the behavioral spectrum in reverse to negative emotions, which are associated with more circumscribed responses (e.g., withdrawal associated with sadness). When it comes to loving kindness and compassion specifically, the action tendencies that follow are those of interactional and interpersonal engagement, even if the emotional responses may differ between the two (happiness versus compassion), thus pointing to their unique contribution in alleviating depression and social anxiety (Hofmann et al., 2011). Metta meditation has the potential to increase positive affect, especially since it does not rely on transient, hedonic values, but fosters a deeper sense of kindness and connectedness (Hofmann et al., 2011, 2012). In this sense, meta-analytic results show that loving kindness increases the level positive emotions, potentially more so the peaceful and prosocial emotions, to a larger extent compared to compassion interventions (Zeng et al., 2015). This is not surprising, given the fact that compassion and self-compassion interventions stem from the experience and acceptance of suffering. Also, in a proof-ofconcept study, loving kindness meditation, administered as a 12-session group intervention, was found to be effective for symptoms of dysthymia and depression, as well as for increasing positive affect (Hofmann et al., 2015).

\section{Including Metta Interventions Into CBT}

Apart from using them as independent interventions, metta interventions can be used adjunctively to CBT protocols in order to increase positive affect levels and to create an appropriate emotional climate for clients to easily accept the cognitive restructuring of negative self-schemas. Metta meditation can be time restricted as to accommodate typical CBT sessions, with the advantage of facilitating cognitive restructuring and potentially also increasing the level of positive affect. Metta interventions can be delivered as exercises in individual and group psychotherapy, practiced in the beginning or at the end of the CBT session, and then also as homework. We can also design interventions which include one or two sessions of metta interventions, then maintain the interventions as homework while the protocol proceeds as usual. Some authors also designed protocols where compassion-based sessions are introduced at the end of treatment, following the first standard CBT sessions (e.g., Asano et al., 2017). However, given that both loving kindness and self-compassion meditation are, like mindfulness, abilities acquired in time, it is essential to practice them continually. Nowadays, we can find various types of loving kindness and self-compassion exercises online, which is a useful resource for both patients and practitioners. Although we believe these interventions can be easily practiced by CBT trained therapists (with no additional credentials), exercising these abilities oneself, as well as advanced reading and practical training are important prerequisites. So 
far, few studies have tested the combination of CBT and metta interventions in clinical trials.

To date, experimental data has shown that adding a preparatory self-compassion exercise prior to a cognitive reappraisal task increases the efficacy of the latter in depressed individuals, thus providing encouraging results (Diedrich, Hofmann, Cuijpers, \& Berking, 2016). That is, participants who practiced a self-compassion exercise (seeing oneself as from outside with a compassionate mindset) showed afterwards a greater reduction in negative emotions during cognitive reappraisal, following a negative mood induction task. These results point to the usefulness of metta interventions in facilitating cognitive restructuring, thus providing another argument for incorporating them into CBT.

Similarly, one of our ongoing studies aims to investigate how group CBT plus positive affect training (including mindfulness and loving kindness meditation) fares for people with low positive mood, irrespective of disorder. In this study, the protocol (12 sessions) introduces the concept and practice of mindfulness as an adjunct to forthcoming loving kindness meditation in the first two sessions, while the concept of loving kindness is introduced and practiced starting with session 3 (approximately 40 minutes). The following group sessions (4-12) begin with a brief 10-minute mindfulness exercise, then classic CBT techniques and exercises (e.g., thought record, cognitive restructuring, behavioral activation) are introduced, and followed by metta meditation (10 minutes) and debriefing (10 minutes), each time promoting a more challenging target of loving kindness (e.g., beloved friend, then someone neutral, etc.). Participants are also instructed to practice the meditation exercises at home.

Other studies have investigated the use of compassion-based interventions (i.e., not only meditation, but also exercises such as letter writing) in combination with CBT. For instance, a single group feasibility study tested the combination of CBT with compassion intervention sessions in depressed participants with good results (Asano et al., 2017). The protocol (10 sessions) used standard CBT and, in the last three sessions approached shame and self-criticism, memories of compassion, and compassion letters. Combining CBT with compassionate mind training in 12 sessions (combining imagery exercises with other techniques, like letter writing and grounding) was also shown to be effective for people referred to therapy as victims of traumatic incidents, with the CBT plus compassionate mind training scoring higher on self-compassion post-therapy (Beaumont et al., 2012).

Finally, we have to keep in mind the fact that some barriers may restrict the usefulness of metta interventions. For instance, individuals high on self-criticism often show a fear of compassion and self-compassion, because these states are experienced as unfamiliar and sometimes, as a sign of weakness (Gilbert, McEwan, Matos, \& Rivis, 2011). Also, precisely because metta interventions foster a sense of calm and soothing rather than a more energetic kind of positive affect, they may appeal less to some people who value the latter kind of emotions more (Galante et al., 2014). In these cases, introducing other 
compassion-based techniques besides meditation could be helpful (e.g., two-chair technique with the critical and the criticized self).

\section{Conclusion}

Metta interventions have been shown to be effective for a wide range of emotional problems, reducing shame and self-criticism, and also increasing positive affect. This allows for conversions into promising interventions especially for disorders characterized by harsh, critical, inflexible self-views and low positive affect, with depressive and social anxiety disorders as the most prevalent. Precisely because loving kindness and compassion are experienced less by these patients, metta interventions appear to be particularly useful; whether they really succeed, however, is still an empirical question (e.g., they may be difficult to practice by these participants again, because they lack these abilities in the first place). More data are needed in clinical populations, as well as for comparisons with active control groups. Nonetheless, so far it seems that metta interventions are effective as independent interventions, as well as emotion regulation strategies, and potentially as adjuncts to CBT protocols as well. Future research should look into the added benefits of combining loving kindness and compassion interventions with established treatment protocols and address their mechanisms of change.

Funding: Dr. Hofmann receives financial support from the Alexander von Humboldt Foundation (as part of the Humboldt Prize), NIH/NCCIH (R01AT007257), NIH/NIMH (R01MH099021, U01MH108168), and the James S.

McDonnell Foundation 21st Century Science Initiative in Understanding Human Cognition - Special Initiative. He receives compensation for his work as editor from SpringerNature and the Association for Psychological Science, and as an advisor from the Palo Alto Health Sciences and for his work as a Subject Matter Expert from John Wiley \& Sons, Inc. and SilverCloud Health, Inc. He also receives royalties and payments for his editorial work from various publishers.

Competing Interests: The authors declare no conflicts of interest.

Acknowledgments: The authors have no support to report.

\section{References}

Arimitsu, K., \& Hofmann, S. G. (2017). Effects of compassionate thinking on negative emotions. Cognition and Emotion, 31(1), 160-167. https://doi.org/10.1080/02699931.2015.1078292

Asano, K., Koike, H., Shinohara, Y., Kamimori, H., Nakagawa, A., Iyo, M., \& Shimizu, E. (2017).

Group cognitive behavioural therapy with compassion training for depression in a Japanese community: A single-group feasibility study. BMC Research Notes, 10(1), Article 670.

https://doi.org/10.1186/s13104-017-3003-0 
Beaumont, E., Galpin, A., \& Jenkins, P. (2012). 'Being kinder to myself ': A prospective comparative study, exploring post-trauma therapy outcome measures, for two groups of clients, receiving either Cognitive Behaviour Therapy or Cognitive Behaviour Therapy and Compassionate Mind Training. Counselling Psychology Review, 27(1), 31-43.

Beck, A. T., \& Alford, B. A. (2009). Depression: Causes and treatment (2nd ed). Philadelphia, PA, USA: University of Pennsylvania Press.

Brown, T. A. (2007). Temporal course and structural relationships among dimensions of temperament and DSM-IV anxiety and mood disorder constructs. fournal of Abnormal Psychology, 116(2), 313-328. https://doi.org/10.1037/0021-843X.116.2.313

Cândea, D.-M., \& Szentágotai-Tătar, A. (2018). The impact of self-compassion on shame-proneness in social anxiety. Mindfulness, 9(6), 1816-1824. https://doi.org/10.1007/s12671-018-0924-1

Carson, J. W., Keefe, F. J., Lynch, T. R., Carson, K. M., Goli, V., Fras, A. M., \& Thorp, S. R. (2005). Loving kindness meditation for chronic low back pain: Results from a pilot trial. Journal of Holistic Nursing, 23(3), 287-304. https://doi.org/10.1177/0898010105277651

Clark, D. M., \& Wells, A. (1995). A cognitive model of social phobia. In R. G. Heimberg, M. R. Liebowitz, D. A. Hope, \& F. R. Schneier (Eds.), Social phobia: Diagnosis, assessment and treatment (pp. 69-93). New York, NY, USA: The Guilford Press.

Cox, B. J., Fleet, C., \& Stein, M. B. (2004). Self-criticism and social phobia in the US national comorbidity survey. Fournal of Affective Disorders, 82(2), 227-234.

https://doi.org/10.1016/j.jad.2003.12.012

Diedrich, A., Burger, J., Kirchner, M., \& Berking, M. (2017). Adaptive emotion regulation mediates the relationship between self-compassion and depression in individuals with unipolar depression. Psychology and Psychotherapy: Theory, Research and Practice, 90(3), 247-263. https://doi.org/10.1111/papt.12107

Diedrich, A., Grant, M., Hofmann, S. G., Hiller, W., \& Berking, M. (2014). Self-compassion as an emotion regulation strategy in major depressive disorder. Behaviour Research and Therapy, 58, 43-51. https://doi.org/10.1016/j.brat.2014.05.006

Diedrich, A., Hofmann, S. G., Cuijpers, P., \& Berking, M. (2016). Self-compassion enhances the efficacy of explicit cognitive reappraisal as an emotion regulation strategy in individuals with major depressive disorder. Behaviour Research and Therapy, 82, 1-10.

https://doi.org/10.1016/j.brat.2016.04.003

Farmer, A. S., Kashdan, T. B., \& Weeks, J. W. (2014). Positivity deficits in social anxiety: Emotions, events, and cognitions. In S. G. Hofmann \& P. M. DiBartolo (Eds.), Social anxiety: Clinical, developmental, and social perspectives (3rd ed.). Oxford, United Kingdom: Elsevier.

Feldman, G., Greeson, J., \& Senville, J. (2010). Differential effects of mindful breathing, progressive muscle relaxation, and loving kindness meditation on decentering and negative reactions to repetitive thoughts. Behaviour Research and Therapy, 48(10), 1002-1011.

https://doi.org/10.1016/j.brat.2010.06.006 
Fergus, T. A., Valentiner, D. P., McGrath, P. B., \& Jencius, S. (2010). Shame- and guilt-proneness: Relationships with anxiety disorder symptoms in a clinical sample. Fournal of Anxiety Disorders, 24(8), 811-815. https://doi.org/10.1016/j.janxdis.2010.06.002

Finlay-Jones, A. L. (2017). The relevance of self-compassion as an intervention target in mood and anxiety disorders: A narrative review based on an emotion regulation framework: Selfcompassion and emotion regulation. Clinical Psychologist, 21(2), 90-103. https://doi.org/10.1111/cp.12131

Flett, G. L, \& Hewitt, P. L. (2014). Perfectionism and perfectionistic self-presentation in social anxiety. In S. G. Hofmann \& P. M. DiBartolo (Eds.), Social anxiety: Clinical, developmental, and social perspectives (3rd ed.). Oxford, United Kingdom: Elsevier.

Fredrickson, B. L. (2001). The role of positive emotions in positive psychology: The broaden-and-build theory of positive emotions. American Psychologist, 56(3), 218-226.

Galante, J., Galante, I., Bekkers, M.-J., \& Gallacher, J. (2014). Effect of kindness-based meditation on health and well-being: A systematic review and meta-analysis. fournal of Consulting and Clinical Psychology, 82(6), 1101-1114. https://doi.org/10.1037/a0037249

Gilbert, P. (2005). Compassion: conceptualisations, research and use in psychotherapy. Retrieved from http://public.eblib.com/choice/publicfullrecord.aspx?p=238730

Gilbert, P., Clarke, M., Hempel, S., Miles, J. N. V., \& Irons, C. (2004). Criticizing and reassuring oneself: An exploration of forms, styles and reasons in female students. British fournal of Clinical Psychology, 43(1), 31-50. https://doi.org/10.1348/014466504772812959

Gilbert, P., McEwan, K., Matos, M., \& Rivis, A. (2011). Fears of compassion: Development of three self-report measures: Fears of compassion. Psychology and Psychotherapy: Theory, Research and Practice, 84(3), 239-255. https://doi.org/10.1348/147608310X526511

Gilbert, P., Pehl, J., \& Allan, S. (1994). The phenomenology of shame and guilt: An empirical investigation. British fournal of Medical Psychology, 67(1), 23-36. https://doi.org/10.1111/j.2044-8341.1994.tb01768.x

Gilbert, P., \& Procter, S. (2006). Compassionate mind training for people with high shame and selfcriticism: Overview and pilot study of a group therapy approach. Clinical Psychology \& Psychotherapy, 13(6), 353-379. https://doi.org/10.1002/cpp.507

Gotlib, I. H., \& Joormann, J. (2010). Cognition and depression: Current status and future directions. Annual Review of Clinical Psychology, 6(1), 285-312. https://doi.org/10.1146/annurev.clinpsy.121208.131305

Graser, J., \& Stangier, U. (2018). Compassion and Loving kindness meditation: An overview and prospects for the application in clinical samples. Harvard Review of Psychiatry, 26(4), 201-215. https://doi.org/10.1097/HRP.0000000000000192

Hayes, S. C. (2004). Acceptance and commitment therapy, relational frame theory, and the third wave of behavioral and cognitive therapies. Behavior Therapy, 35(4), 639-665. https://doi.org/10.1016/S0005-7894(04)80013-3 
Hofmann, S. G. (2007). Cognitive factors that maintain social anxiety disorder: A comprehensive model and its treatment implications. Cognitive Behaviour Therapy, 36(4), 193-209. https://doi.org/10.1080/16506070701421313

Hofmann, S. G. (2014). Interpersonal emotion regulation model of mood and anxiety disorders. Cognitive Therapy and Research, 38, 483-492. https://doi.org/10.1007/s10608-014-9620-1

Hofmann, S. G. (2016). Emotion in therapy: From science to practice. New York, NY, USA: Guilford Press.

Hofmann, S. G., Carpenter, J. K., \& Curtiss, J. (2016). Interpersonal Emotion Regulation Questionnaire (IERQ): Scale development and psychometric characteristics. Cognitive Therapy and Research, 40, 341-356. https://doi.org/10.1007/s10608-016-9756-2

Hofmann, S. G., \& Doan, S. N. (2018). The social foundations of emotion: Developmental, cultural, and clinical dimensions. Washington, DC, USA: American Psychological Association.

Hofmann, S. G., Grossman, P., \& Hinton, D. E. (2011). Loving kindness and compassion meditation: Potential for psychological interventions. Clinical Psychology Review, 31(7), 1126-1132. https://doi.org/10.1016/j.cpr.2011.07.003

Hofmann, S. G., \& Otto, M. W. (2018). Cognitive behavioral therapy for social anxiety disorder: Evidence-based and disorder-specific treatment techniques (2nd ed.). Abingdon, United Kingdom: Routledge.

Hofmann, S. G., Petrocchi, N., Steinberg, J., Lin, M., Arimitsu, K., Kind, S., . . Stangier, U. (2015). Loving-kindness meditation to target affect in mood disorders: A proof-of-concept study. Evidence-Based Complementary and Alternative Medicine, 2015, Article 269126.

Hofmann, S. G., Sawyer, A. T., Fang, A., \& Asnaani, A. (2012). Emotion Dysregulation Model of Mood and Anxiety Disorders. Depression and Anxiety, 29(5), 409-416. https://doi.org/10.1002/da.21888

Hofmann, S. G., Sawyer, A. T., Witt, A. A., \& Oh, D. (2010). The effect of mindfulness-based therapy on anxiety and depression: A meta-analytic review. fournal of Consulting and Clinical Psychology, 78(2), 169-183. https://doi.org/10.1037/a0018555

Hutcherson, C. A., Seppala, E. M., \& Gross, J. J. (2008). Loving kindness meditation increases social connectedness. Emotion, 8(5), 720-724. https://doi.org/10.1037/a0013237

Iancu, I., Bodner, E., \& Ben-Zion, I. Z. (2015). Self esteem, dependency, self-efficacy and selfcriticism in social anxiety disorder. Comprehensive Psychiatry, 58, 165-171. https://doi.org/10.1016/j.comppsych.2014.11.018

Jazaieri, H., McGonigal, K., Jinpa, T., Doty, J. R., Gross, J. J., \& Goldin, P. R. (2014). A randomized controlled trial of compassion cultivation training: Effects on mindfulness, affect, and emotion regulation. Motivation and Emotion, 38(1), 23-35. https://doi.org/10.1007/s11031-013-9368-z

Kabat-Zinn, J. (1982). An outpatient program in behavioral medicine for chronic pain patients based on the practice of mindfulness meditation: Theoretical considerations and preliminary results. General Hospital Psychiatry, 4(1), 33-47. https://doi.org/10.1016/0163-8343(82)90026-3 
Kirby, J. N. (2017). Compassion interventions: The programmes, the evidence, and implications for research and practice. Psychology and Psychotherapy: Theory, Research and Practice, 90(3), 432-455. https://doi.org/10.1111/papt.12104

Krieger, T., Altenstein, D., Baettig, I., Doerig, N., \& Holtforth, M. G. (2013). Self-compassion in depression: Associations with depressive symptoms, rumination, and avoidance in depressed outpatients. Behavior Therapy, 44(3), 501-513. https://doi.org/10.1016/j.beth.2013.04.004

Krieger, T., Berger, T., \& Holtforth, M. G. (2016). The relationship of self-compassion and depression: Cross-lagged panel analyses in depressed patients after outpatient therapy. fournal of Affective Disorders, 202, 39-45. https://doi.org/10.1016/j.jad.2016.05.032

Krieger, T., Reber, F., von Glutz, B., Urech, A., Moser, C. T., Schulz, A., \& Berger, T. (2019). An internet-based compassion-focused intervention for increased self-criticism: A randomized controlled trial. Behavior Therapy, 50(2), 430-445. https://doi.org/10.1016/j.beth.2018.08.003

Linehan, M. M., Schmidt, H., Dimeff, L. A., Craft, J. C., Kanter, J., \& Comtois, K. A. (1999). Dialectical behavior therapy for patients with borderline personality disorder and drugdependence. American fournal on Addictions, 8(4), 279-292. https://doi.org/10.1080/105504999305686

López, A., Sanderman, R., \& Schroevers, M. J. (2018). A close examination of the relationship between self-compassion and depressive symptoms. Mindfulness, 9(5), 1470-1478. https://doi.org/10.1007/s12671-018-0891-6

Luyten, P., Sabbe, B., Blatt, S. J., Meganck, S., Jansen, B., De Grave, C., . . Corveleyn, J. (2007). Dependency and self-criticism: Relationship with major depressive disorder, severity of depression, and clinical presentation. Depression and Anxiety, 24(8), 586-596. https://doi.org/10.1002/da.20272

MacBeth, A., \& Gumley, A. (2012). Exploring compassion: A meta-analysis of the association between self-compassion and psychopathology. Clinical Psychology Review, 32(6), 545-552. https://doi.org/10.1016/j.cpr.2012.06.003

Mascaro, J. S., Rilling, J. K., Tenzin Negi, L., \& Raison, C. L. (2013). Compassion meditation enhances empathic accuracy and related neural activity. Social Cognitive and Affective Neuroscience, 8(1), 48-55. https://doi.org/10.1093/scan/nss095

Mongrain, M., \& Leather, F. (2006). Immature dependence and self-criticism predict the recurrence of major depression. Journal of Clinical Psychology, 62(6), 705-713. https://doi.org/10.1002/jclp.20263

Neff, K. (2003). Self-compassion: An alternative conceptualization of a healthy attitude toward oneself. Self and Identity, 2(2), 85-101. https://doi.org/10.1080/15298860309032

Neff, K. (2011). Self-compassion: Stop beating yourself up and leave insecurity behind. New York, NY, USA: William Morrow.

Segal, Z. V., Williams, J. M. G., \& Teasdale, J. D. (2002). Mindfulness-based cognitive therapy for depression: A new approach to preventing relapse. New York, NY, USA: Guilford Press. 
Shonin, E., Van Gordon, W., Compare, A., Zangeneh, M., \& Griffiths, M. D. (2015). Buddhist-derived loving-kindness and compassion meditation for the treatment of psychopathology: A systematic review. Mindfulness, 6(5), 1161-1180. https://doi.org/10.1007/s12671-014-0368-1

Thompson, B. L., \& Waltz, J. (2008). Self-compassion and PTSD symptom severity. fournal of Traumatic Stress, 21(6), 556-558. https://doi.org/10.1002/jts.20374

Visted, E., Vøllestad, J., Nielsen, M. B., \& Schanche, E. (2018). Emotion regulation in current and remitted depression: A systematic review and meta-analysis. Frontiers in Psychology, 9, Article 756. https://doi.org/10.3389/fpsyg.2018.00756

Werner, K. H., Jazaieri, H., Goldin, P. R., Ziv, M., Heimberg, R. G., \& Gross, J. J. (2012). Selfcompassion and social anxiety disorder. Anxiety, Stress \& Coping, 25(5), 543-558.

https://doi.org/10.1080/10615806.2011.608842

Zeng, X., Chiu, C. P. K., Wang, R., Oei, T. P. S., \& Leung, F. Y. K. (2015). The effect of lovingkindness meditation on positive emotions: a meta-analytic review. Frontiers in Psychology, 6, Article 1693. https://doi.org/10.3389/fpsyg.2015.01693

\section{EACLIPT}

Clinical Psychology in Europe (CPE) is the official journal of the European Association of Clinical Psychology and Psychological Treatment (EACLIPT).

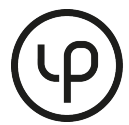

leibniz-psychology.org

PsychOpen GOLD is a publishing service by Leibniz Institute for Psychology Information (ZPID), Germany. 